\title{
Wavefield Focusing in Finite Time with Reduced Spatial Exposure
}

\author{
Giovanni Angelo Meles ${ }^{1}$, Joost van der Neut ${ }^{2}$, Koen W. A. van Dongen ${ }^{2}$ and Kees Wapenaar ${ }^{1}$
}

July 3, 2021

\footnotetext{
${ }^{1}$ Faculty of Civil Engineering \& Geosciences, Delft University of Technology, Delft, The Netherlands

${ }^{2}$ Faculty of Applied Sciences, Delft University of Technology, Delft, The Netherlands

G.A.Meles@tudelft.nl
}

\section{$\infty 1$ Abstract}

Wavefield focusing is often achieved by time-reversal mirrors, which are typically derived from closed-boundary integral representations. In heterogeneous media, time-reversal focusing theoretically involves both in- and output signals that are infinite in time and where the resulting waves propagate through the entire medium. Recently, new integral representations have been derived for single-sided wavefield focusing. Although the required input signals for this approach are finite in time, the output signals are not and, similarly to timereversal mirroring, the waves propagate through the entire medium. Here, an alternative solution for doublesided wavefield focusing is derived. In this solution, based on a new integral representation where both inand output signals are finite in time, the energy of the waves propagate in the layer embedding the focal point is smaller than with time-reversal focusing. This approach has therefore the potential to reduce spatial and temporal exposure in wavefield focusing experiments, as well as to aid to synthetic wavefield focusing for imaging and inversion. We explore the potential of the new method with numerical experiments involving a simplified head model consisting of a skull enclosing a brain.

\section{Introduction}

With time-reversal mirrors, wavefields can be focused at a specified focal point in an arbitrary heterogeneous medium [1]. To realize such a mirror, wavefields from a source at the focal point are evaluated at a closed boundary and sent back, after time-reversal, into the medium from that boundary. As can be demonstrated from Green's theorem, this procedure leads to a solution of the homogeneous wave equation, consisting of an acausal wavefield that focuses at the focal point and a causal wavefield, propagating from the focal point through the entire medium to the boundary [2]. Applications can be found in various areas. In medical acoustics, the time-reversal mirror has been applied for kidney stone and tumor ablation [3, 4]. The time-reversal concept is also a key ingredient for various source localization [5, 6] and reflection imaging [7, 8, algorithms. Assuming that the medium is lossless and sufficiently heterogeneous, both the acausal wavefield that propagates towards the focal point and the causal wavefield that propagates through the medium to the boundary are unbounded in time.

Recently, it was shown that wavefields in one-dimensional media can also be focused from a single openboundary by solving the Marchenko equation [9], being a familiar result from inverse scattering theory [10. In this case a different focusing condition is achieved [11], and when the solution of the Marchenko equation is emitted into the medium, a focus emerges at the focal point and a causal Green's function propagates through the entire medium to the boundary [12]. This result can be extended to three-dimensional wave propagation [13] and has seen various applications in exploration geophysics, such as reflection imaging [14] and acoustic holography [15]. Although the focusing function is finite in time, the Green's function that emerges after wavefield focusing has infinite duration. In this paper, it will be shown how a focusing wavefield can be constructed that propagates to a specified focal point in finite time with reduced wavefield propagation in the layer embedding the focal point. 


\section{Theory}

Coordinates in three-dimensional space are defined as $\mathbf{x}=\left(x_{1}, x_{2}, x_{3}\right)$, and $t$ denotes time. Although the derived theory can be modified for various types of wave phenomena, acoustic wave propagation is considered. The medium is lossless and characterized by propagation velocity $c(\mathbf{x})$ and mass density $\rho(\mathbf{x})$. It is assumed that these properties are independent of time. The acoustic pressure wavefield is expressed as $p(\mathbf{x}, t)$. The temporal Fourier transform of $p(\mathbf{x}, t)$ is defined by $p(\mathbf{x}, \omega)=\int_{-\infty}^{\infty} p(\mathbf{x}, t) \exp (-j \omega t) d t$, where $\omega$ is the angular frequency. All wavefields obey the wave equation, which is defined in the frequency domain as

$$
\partial_{i}\left(\frac{1}{\rho(\mathbf{x})} \partial_{i} p(\mathbf{x}, \omega)\right)+\frac{\omega^{2}}{\rho(\mathbf{x}) \mathrm{c}^{2}(\mathbf{x})} p(\mathbf{x}, \omega)=-j \omega q(\mathbf{x}, \omega),
$$

with spatial derivatives $\partial_{i}=\frac{\partial}{\partial x_{i}}$, where $i$ takes the values 1,2 and 3 . Einstein's summation convention is applied, meaning that summation is carried out over repeated indeces. Note that the source function $q(\mathbf{x}, \omega)$, standing for volume-injection rate density, is scaled by $j \omega$. Since the wave equation is often defined without this scaling factor elsewhere in the literature, the wavefields that appear in this paper should be divided with $(j \omega)$ to be consistent with that literature. The Green's function $G\left(\mathbf{x}, \mathbf{x}_{S}, \omega\right)$ is defined as the solution of the wave equation for $q(\mathbf{x}, \omega)=\delta\left(\mathbf{x}-\mathbf{x}_{S}\right)$, where $\mathbf{x}_{S}$ is the source location.

It has been shown how the real part of the Green's function with a source at $\mathbf{x}_{A}$ and a receiver at $\mathbf{x}_{B}$ can be expressed by integrating a specific combination of observations from a source at $\mathbf{x}_{A}$ and a source at $\mathbf{x}_{B}$ over any boundary $\partial \mathbb{D}$ that encloses volume $\mathbb{D}$, where $\mathbf{x}_{A} \in \mathbb{D}$ and $\mathbf{x}_{B} \in \mathbb{D}$ (Fig. 17):

$$
2 \Re\left\{G\left(\mathbf{x}_{B}, \mathbf{x}_{A} ; \omega\right)\right\}=\oint_{\partial \mathbb{D}} d^{2} \mathbf{x} \frac{1}{j \omega \rho(\mathbf{x})}\left(G\left(\mathbf{x}, \mathbf{x}_{B}, \omega\right) n_{i} \partial_{i} G^{*}\left(\mathbf{x}, \mathbf{x}_{A}, \omega\right)-G^{*}\left(\mathbf{x}, \mathbf{x}_{A}, \omega\right) n_{i} \partial_{i} G\left(\mathbf{x}, \mathbf{x}_{B}, \omega\right)\right),
$$

where $n_{i}$ is the outward pointing normal of $\partial \mathbb{D}$ and superscript $*$ denotes complex conjugation. In time-reversed acoustics, observations from a source at $\mathbf{x}_{A}$ are reversed in time and injected into the medium at $\partial \mathbb{D}$. The result of this approach can be interpreted as if the injected field were propagated forward in time to any location $\mathbf{x}_{B}$ by the Green's function $G\left(\mathbf{x}_{B}, \mathbf{x}, \omega\right)$, which can be related to $G\left(\mathbf{x}, \mathbf{x}_{B}, \omega\right)$ through source-receiver reciprocity [16]. As can be learned from Eq. 2, this procedure yields for any location $\mathbf{x}_{B}$ the real part of the Green's function $G\left(\mathbf{x}_{B}, \mathbf{x}_{A} ; \omega\right)$, which can be interpreted as the Fourier transform of the superposition of an acausal Green's function, focusing at $\mathbf{x}=\mathbf{x}_{A}$, and a causal Green's function that propagates through the entire medium to $\partial \mathbb{D}$. Since the source functions of this acausal and causal Green's function cancel each other, their superposition satisfies the homogeneous wave equation (i.e. Eq. 1 for $q(\mathbf{x}, \omega)=0$ ). Note that this homogeneous wave equation is valid also for heterogeneous media. Note also that time-reversed acoustics results in a wavefield that at time $t=0$ is non-zero just at the focal point [17], but it poses no constraints on the wavefield at other times.

We also consider a peculiar closed boundary $\partial \mathbb{D}=\partial \mathbb{D}_{1} \cup \partial \mathbb{D}_{2} \cup \partial \mathbb{D}_{\text {cyl }}$, where $\partial \mathbb{D}_{1}$ and $\partial \mathbb{D}_{2}$ are horizontal boundaries connected by a cylindrical surface $\partial \mathbb{D}_{c y l}$ with infinite radius (Fig. 1 b). For this configuration, the contribution of the integral in Eq. 2 over $\partial \mathbb{D}_{c y l}$ vanishes and the following representation holds [18]:

$$
2 \Re\left\{G\left(\mathbf{x}_{B}, \mathbf{x}_{A} ; \omega\right)\right\}=\int_{\partial \mathbb{D}_{1} \cup \partial \mathbb{D}_{2}} d^{2} \mathbf{x} \frac{1}{j \omega \rho(\mathbf{x})}\left(G\left(\mathbf{x}, \mathbf{x}_{B}, \omega\right) n_{3} \partial_{3} G^{*}\left(\mathbf{x}, \mathbf{x}_{A}, \omega\right)-G^{*}\left(\mathbf{x}, \mathbf{x}_{A}, \omega\right) n_{3} \partial_{3} G\left(\mathbf{x}, \mathbf{x}_{B}, \omega\right)\right) .
$$

In addition to standard time-reversed acoustics, interesting focusing wavefields can be derived also by using focusing functions, which have recently been introduced to denote the solutions of the multidimensional Marchenko equation [13]. In this derivation, the same horizontal boundaries $\partial \mathbb{D}_{1}$ and $\partial \mathbb{D}_{2}$ as in Eq. 3 are used, but an additional auxiliary boundary $\partial \mathbb{D}_{A}$ is introduced. Here, $\partial \mathbb{D}_{A}$ is a horizontal plane inside $\mathbb{D}$ that intersects with the focal point $\mathbf{x}_{A}=\left(x_{1, A}, x_{2, A}, x_{3, A}\right)$, so that volume $\mathbb{D}$ is divided into a subvolume $\mathbb{D}_{1}$, located above $\partial \mathbb{D}_{A}$, and a subvolume $\mathbb{D}_{2}$, located below $\partial \mathbb{D}_{A}$ (Fig. 1 f). Note that the normals along $\partial \mathbb{D}_{A}$ associated with subvolumes $\mathbb{D}_{1}$ and $\mathbb{D}_{2}$ are antiparallel (Fig. 1 c).

We deduce new sets of representation theorems for volumes $\mathbb{D}_{1}$ and $\mathbb{D}_{2}$. First of all, a reciprocity theorem of the convolution type [16] associated with volume $\mathbb{D}_{1}$ is introduced: 


$$
\int_{\mathbb{D}_{1}} d^{3} \mathbf{x}\left(p_{A} q_{B}-p_{B} q_{A}\right)=\int_{\partial \mathbb{D}_{1}} d^{2} \mathbf{x} \frac{1}{j \omega \rho}\left(p_{B} n_{3} \partial_{3} p_{A}-p_{A} n_{3} \partial_{3} p_{B}\right)-\int_{\partial \mathbb{D}_{A}} d^{2} \mathbf{x} \frac{2}{j \omega \rho}\left(p_{A}^{+} \partial_{3} p_{B}^{-}+p_{A}^{-} \partial_{3} p_{B}^{+}\right) .
$$

Subscripts $A$ and $B$ indicate two states. The integral over $\partial \mathbb{D}_{A}$ has been modified by using fundamental properties [19] of the (Helmholtz) operator in Eq. 2, where the wavefields have been decomposed into downgoing (indicated by superscript + ) and upgoing (indicated by superscript -) constituents. In addition, the field has been normalized such that $p=p^{+}+p^{-}$. Similarly, a reciprocity theorem of the correlation type [20] can be modified as

$$
\int_{\mathbb{D}_{1}} d^{3} \mathbf{x}\left(p_{A}^{*} q_{B}+p_{B} q_{A}^{*}\right)=\int_{\partial \mathbb{D}_{1}} d^{2} \mathbf{x} \frac{1}{j \omega \rho}\left(p_{B} n_{3} \partial_{3} p_{A}^{*}-p_{A}^{*} n_{3} \partial_{3} p_{B}\right)-\int_{\partial \mathbb{D}_{A}} d^{2} \mathbf{x} \frac{2}{j \omega \rho}\left(p_{A}^{+*} \partial_{3} p_{B}^{+}+p_{A}^{-*} \partial_{3} p_{B}^{-}\right) .
$$

Two representations will be derived for subvolume $\mathbb{D}_{1}$. In both representations, state $A$ is source-free $\left(q_{A}=0\right)$. The medium properties in this state are identical to the physical properties $c(\mathbf{x})$ and $\rho(\mathbf{x})$ within $\mathbb{D}_{1}$, but chosen such that the halfspace below $\partial \mathbb{D}_{A}$ is non-reflective. A particular solution of the source-free wave equation will be substituted in this state, which is referred to as focusing function $p_{A}=f_{1}\left(\mathbf{x}, \mathbf{x}_{A}, \omega\right)$, where $\mathbf{x}_{A}$ is the focal point and $\mathbf{x}$ is a variable coordinate inside the domain $\mathbb{D}[13$. This focusing function is subject to a different focusing condition than what is achieved by time-reversed acoustics. In this paper, this condition is defined slightly different than in aforementioned literature as $\left.f_{1}^{+}\left(\mathbf{x}, \mathbf{x}_{A} ; \omega\right)\right|_{\mathbf{x} \in \partial \mathbb{D}_{A}}=\delta\left(\mathbf{x}_{H}-\mathbf{x}_{H, A}\right)$, where $\mathbf{x}_{H}=\left(x_{1}, x_{2}\right)$, while $\left.f_{1}^{-}\left(\mathbf{x}, \mathbf{x}_{A} ; \omega\right)\right|_{\mathbf{x} \in \partial \mathbb{D}_{A}}$ vanishes. The first condition states that the downgoing part of the focusing function focuses at $\mathbf{x}_{A}$. After having focused, this downgoing function continues its propagation into the non-reflective lower half-space, which explains why the upgoing part of the focusing function at $\partial \mathbb{D}_{A}$ is zero. Note that this condition does not pose any constraint on the wavefield at time $t=0$ away from the focal plane $\partial \mathbb{D}_{A}$. In state $B$, the medium properties are equivalent to the physical medium, where an impulsive source is located at $\mathbf{x}_{B} \in \mathbb{D}$, yielding $q_{B}=\delta\left(\mathbf{x}-\mathbf{x}_{B}\right)$ and $p_{B}=G\left(\mathbf{x}, \mathbf{x}_{B} ; \omega\right)$. Substituting these quantities into Eqs. 4 and 5 brings

$$
\begin{array}{r}
\theta\left(x_{3, A}-x_{3, B}\right) f_{1}\left(\mathbf{x}_{B}, \mathbf{x}_{A} ; \omega\right)+\frac{2}{j \omega \rho\left(\mathbf{x}_{\mathbf{A}}\right)} \partial_{3} G^{-}\left(\mathbf{x}_{A}, \mathbf{x}_{B}, \omega\right)=\int_{\partial \mathbb{D}_{1}} d^{2} \mathbf{x} \frac{1}{j \omega \rho(\mathbf{x})} \times \\
\left(G\left(\mathbf{x}, \mathbf{x}_{B}, \omega\right) n_{3} \partial_{3} f_{1}\left(\mathbf{x}, \mathbf{x}_{A}, \omega\right)-f_{1}\left(\mathbf{x}, \mathbf{x}_{A}, \omega\right) n_{3} \partial_{3} G\left(\mathbf{x}, \mathbf{x}_{B}, \omega\right)\right),
\end{array}
$$

and

$$
\begin{array}{r}
\theta\left(x_{3, A}-x_{3, B}\right) f_{1}^{*}\left(\mathbf{x}_{B}, \mathbf{x}_{A} ; \omega\right)+\frac{2}{j \omega \rho\left(\mathbf{x}_{\mathbf{A}}\right)} \partial_{3} G^{+}\left(\mathbf{x}_{A}, \mathbf{x}_{B}, \omega\right)=\int_{\partial \mathbb{D}_{1}} d^{2} \mathbf{x} \frac{1}{j \omega \rho(\mathbf{x})} \times \\
\left(G\left(\mathbf{x}, \mathbf{x}_{B}, \omega\right) n_{3} \partial_{3} f_{1}^{*}\left(\mathbf{x}, \mathbf{x}_{A}, \omega\right)-f_{1}^{*}\left(\mathbf{x}, \mathbf{x}_{A}, \omega\right) n_{3} \partial_{3} G\left(\mathbf{x}, \mathbf{x}_{B}, \omega\right)\right)
\end{array}
$$

where $\theta\left(x_{3}\right)$ is a Heaviside function, with $\theta\left(x_{3}\right)=0$ for $x_{3}<0, \theta\left(x_{3}\right)=\frac{1}{2}$ for $x_{3}=0$ and $\theta\left(x_{3}\right)=1$ for $x_{3}>0$.

Convolution and correlation theorems associated with volume $\mathbb{D}_{2}$ are also introduced:

$$
\begin{aligned}
\int_{\mathbb{D}_{2}} d^{3} \mathbf{x}\left(p_{A} q_{B}-p_{B} q_{A}\right) & =\int_{\partial \mathbb{D}_{2}} d^{2} \mathbf{x} \frac{1}{j \omega \rho}\left(p_{B} n_{3} \partial_{3} p_{A}-p_{A} n_{3} \partial_{3} p_{B}\right)+\int_{\partial \mathbb{D}_{A}} d^{2} \mathbf{x} \frac{2}{j \omega \rho}\left(p_{A}^{+} \partial_{3} p_{B}^{-}+p_{A}^{-} \partial_{3} p_{B}^{+}\right) \\
\int_{\mathbb{D}_{2}} d^{3} \mathbf{x}\left(p_{A}^{*} q_{B}+p_{B} q_{A}^{*}\right) & =\int_{\partial \mathbb{D}_{2}} d^{2} \mathbf{x} \frac{1}{j \omega \rho}\left(p_{B} n_{3} \partial_{3} p_{A}^{*}-p_{A}^{*} n_{3} \partial_{3} p_{B}\right)+\int_{\partial \mathbb{D}_{A}} d^{2} \mathbf{x} \frac{2}{j \omega \rho}\left(p_{A}^{+*} \partial_{3} p_{B}^{+}+p_{A}^{-*} \partial_{3} p_{B}^{-}\right) .
\end{aligned}
$$

Two representations can be derived for subvolume $\mathbb{D}_{2}$. For both representations, state $A$ is source-free $\left(q_{A}=0\right)$, with medium properties as in the physical state in $\mathbb{D}_{2}$ and a non-reflective halfspace above $\partial \mathbb{D}_{A}$. Focusing function $p_{A}=f_{2}\left(\mathbf{x}, \mathbf{x}_{A}, \omega\right)$ will be substituted, being a solution of the source-free wave equation, with the focusing condition $\left.f_{2}^{-}\left(\mathbf{x}, \mathbf{x}_{A} ; \omega\right)\right|_{\mathbf{x} \in \partial \mathbb{D}_{A}}=\delta\left(\mathbf{x}_{H}-\mathbf{x}_{H, A}\right)$, while $\left.f_{2}^{+}\left(\mathbf{x}, \mathbf{x}_{A} ; \omega\right)\right|_{\mathbf{x} \in \partial \mathbb{D}_{A}}$ vanishes. One again, this focusing function is slightly modified with respect to aforementioned literature [13]. In state $B$, conditions are the same as in the derivation of the previous representations. Substituting these quantities into Eq. 8 and Eq. 9 yields 


$$
\begin{array}{r}
\theta\left(x_{3, B}-x_{3, A}\right) f_{2}\left(\mathbf{x}_{B}, \mathbf{x}_{A} ; \omega\right)-\frac{2}{j \omega \rho\left(\mathbf{x}_{\mathbf{A}}\right)} \partial_{3} G^{+}\left(\mathbf{x}_{A}, \mathbf{x}_{B}, \omega\right)=\int_{\partial \mathbb{D}_{2}} d^{2} \mathbf{x} \frac{1}{j \omega \rho(\mathbf{x})} \times \\
\left(G\left(\mathbf{x}, \mathbf{x}_{B}, \omega\right) n_{3} \partial_{3} f_{2}\left(\mathbf{x}, \mathbf{x}_{A}, \omega\right)-f_{2}\left(\mathbf{x}, \mathbf{x}_{A}, \omega\right) n_{3} \partial_{3} G\left(\mathbf{x}, \mathbf{x}_{B}, \omega\right)\right)
\end{array}
$$

and

$$
\begin{array}{r}
\theta\left(x_{3, B}-x_{3, A}\right) f_{2}^{*}\left(\mathbf{x}_{B}, \mathbf{x}_{A} ; \omega\right)-\frac{2}{j \omega \rho\left(\mathbf{x}_{\mathbf{A}}\right)} \partial_{3} G^{-}\left(\mathbf{x}_{A}, \mathbf{x}_{B}, \omega\right)=\int_{\partial \mathbb{D}_{2}} d^{2} \mathbf{x} \frac{1}{j \omega \rho(\mathbf{x})} \times \\
\left(G\left(\mathbf{x}, \mathbf{x}_{B}, \omega\right) n_{3} \partial_{3} f_{2}^{*}\left(\mathbf{x}, \mathbf{x}_{A}, \omega\right)-f_{2}^{*}\left(\mathbf{x}, \mathbf{x}_{A}, \omega\right) n_{3} \partial_{3} G\left(\mathbf{x}, \mathbf{x}_{B}, \omega\right)\right) .
\end{array}
$$

Note that the different orientation of the normals along $\partial \mathbb{D}_{A}$ when associated with subvolumes $\mathbb{D}_{1}$ or $\mathbb{D}_{2}$ results in opposite signs of the Green's functions terms in Eqs. 6r7 and 10, 11, respectively. Therefore, when Eq. 6, 7 , 10 and 11 are added together, the Green's functions terms cancel out and it follows that:

$$
\begin{aligned}
& 2 \Re\left\{f\left(\mathbf{x}_{B}, \mathbf{x}_{A} ; \omega\right)\right\} \\
& =\int_{\partial \mathbb{D}_{1} \cup \mathbb{D}_{2}} d^{2} \mathbf{x} \frac{1}{j \omega \rho(\mathbf{x})}\left(G\left(\mathbf{x}, \mathbf{x}_{B}, \omega\right) n_{3} \partial_{3} 2 \Re\left\{f\left(\mathbf{x}, \mathbf{x}_{A}, \omega\right)\right\}-2 \Re\left\{f\left(\mathbf{x}, \mathbf{x}_{A}, \omega\right)\right\} n_{3} \partial_{3} G\left(\mathbf{x}, \mathbf{x}_{B}, \omega\right)\right),
\end{aligned}
$$

where

$$
f\left(\mathbf{x}, \mathbf{x}_{A} ; \omega\right)=\theta\left(\mathbf{x}_{3, A}-\mathbf{x}_{3}\right) f_{1}\left(\mathbf{x}, \mathbf{x}_{A} ; \omega\right)+\theta\left(\mathbf{x}_{3}-\mathbf{x}_{3, A}\right) f_{2}\left(\mathbf{x}, \mathbf{x}_{A} ; \omega\right) .
$$

Akin to Eq. 2, this result can be used for wavefield focusing. By injecting wavefield $f\left(\mathbf{x}, \mathbf{x}_{A} ; \omega\right)$, as defined by Eq. 13 , into the medium at boundaries $\partial \mathbb{D}_{1}$ and $\partial \mathbb{D}_{2}$, one can reconstruct this wavefield throughout the volume, as shown by Eq. 12. This wavefield propagates towards the focal point in finite time and back to the surface in finite time again. Moreover, due to the focusing properties of $f_{1}$ and $f_{2}$, the wavefield $f$ theoretically interacts with focal plane $\partial \mathbb{D}_{A}$ only at $\mathbf{x}=\mathbf{x}_{H, A}$ at $t=0$. We refer to the focusing achieved by Eq. 12 as 'focusing in finite time with reduced spatial exposure', which we will often abbreviate as 'focusing in finite time'.

\section{$4 \quad$ Numerical Examples}

For illustration purposes, the right-hand sides of Eqs. 2, 3 and 12 are computed in a two-dimensional layered medium (Fig. 2(a)). The focusing function $f_{1}$ is retrieved using a standard Marchenko configuration [21], with reflection data collected along the upper boundary of the model $\left(\partial \mathbb{D}_{1}\right.$ in Fig. $\left.2(a)\right)$. Similarly, the focusing function $f_{2}$ is retrieved using reflection data collected along the lower boundary of the model $\left(\partial \mathbb{D}_{2}\right.$ in Fig. 2(a)). Initial focusing functions with a $0.8 \mathrm{MHz}$ Ricker wavelet emanating from the focal point (red star in Fig. 2(b)) to the upper and the lower boundaries receivers are used (Fig. 2(b)). The solution from Eq. 2 and 3 has infinite support in time, which could be disadvantageous for various applications. Since the focusing functions $f_{1}$ and $f_{2}$ are confined in time and space by the direct propagation path from the boundary to the focal point [10], so is their superposition $f$. Hence, this solution seems preferable for wavefield focusing in finite time. The focusing function $f$ contains a series of wavefronts that are emitted into the medium from the upper and lower boundaries, and only the first of these wavefronts reaches the focal point. The remaining events are encoded such that any ingoing reflection of the first wavefront is canceled. The focusing conditions satisfied by time-reversed acoustics and finite time focusing differ drastically with respect to wavefield propagation in the focal plane. While in time-reversed acoustics no constraint is posed on the propagation along the focal plane before or after time $t=0$, focusing in finite time limits the interaction of the wavefield with the focal plane at the focal point and at time $t=0$ only. In a two-dimensional experiment we can also explore the spatial properties of focusing in finite time throughout time.

We illustrate this by showing propagation snapshots associated with the right-hand sides of Eqs. 2,3 and 12 . Note that for the sake of brevity in the following we only focus on positive times, but identical considerations apply for the acausal components of the considered wavefields. In time reversed acoustics, the superposition of an acausal and a causal Green's function focusing and propagating away from $\mathbf{x}=\mathbf{x}_{A}$, is expected (Eq. 2 and 
3). Propagation around the foci is perfectly isotropic when Eq. 2 is used (yellow arrows in Fig. 3), while the solution of Eq. 3 results in spurious events (black arrows in Fig. 4) and artefacts, especially in the estimates of the direct wavefield along the focal plane (compare the amplitude of the wavefronts indicated by the yellow arrows in Figs. 3 and 47). These low amplitude artefacts are due to the finite extent of the horizontal boundaries employed in our numerical experiment when Eq. 3 is considered [17]. Note that in any case reflections propagating through the focal plane are well recovered both by Eqs. 2 and 3 (red arrows in Figs. 3 and 4 ). In finite time focusing, destructive interference of up- and down-going wavefields prevents primary as well as multiple reflections to propagate through the focal plane at any time (blue arrows in Fig. 5p. The interaction of the wavefield with the layer embedding the focal point is therefore limited to the propagation of the direct components of $f$. Note that no direct or scattered waves propagating from and to the acquisition surfaces interact with the focal plane except that at the focal point.

The theory and methodology presented here hold also for laterally invariant models, and we show this by applying our focusing strategy to a second numerical experiment. In this case we consider a model consisting of a slice of a human head (see Fig. 6 and Table) and explore the applicability of the method to medical imaging/treatment. This second example is particularly challenging for Marchenko focusing due to the presence of thin layers, diffractors and dipping layers [13. As for the previous example, the focusing functions $f_{1}$ and $f_{2}$ are retrieved using a standard Marchenko configuration, with reflection data collected along the upper and the lower boundaries of the model. Initial focusing functions with a $0.8 \mathrm{MHz}$ Ricker wavelet emanating from the focal point (red star in Fig. 66) to receivers at the upper and the lower boundaries are used. Note that for this example the initial focusing functions are computed in the true model.

We first compare the focusing properties of solutions of Eqs. 3 and 12 by showing snapshots of the corresponding wavefields. Note that as for the previous numerical experiment, also here we only focus on positive times, but identical considerations apply for the acausal components of the considered wavefields. In time reversed acoustics, the superposition of an acausal and a causal Green's function focusing and propagating away from $\mathbf{x}=\mathbf{x}_{A}$, is expected. However, due to the employed truncated boundaries, low amplitude artefacts occurring at time $t=0$ contaminate the wavefield throughout the domain, especially in the proximity of the focal point (red arrows in Fig. 7(a)). Similar artefacts also contaminate the wavefield associated with Eq. 12 (red arrows in Fig. 7(b)). In Figs. 7(c) and 7(e) the wavefield associated with Eq. 3 is shown to propagate almost isotropically around the focal point. More precisely, direct components of the wavefield, interferometrically associated with laterally scattered waves [22, interact with the focal plane (yellow arrow in Fig. 7(c)) at positive times. By contrast, the wavefield associated with Eq. 12 does not exhibit similar components (yellow arrows in Figs. 7(d) and 7(f)). The red arrow in Fig. 7(e) indicates a primary reflection associated with the wall of the skull above the focal plane. As expected, the interaction of the coda of the focusing function (black arrows in Figs. 7(b) and 7(d)) interferes destructively with this reflection (blue arrow in Fig. 7(f)). The cancellation of the ingoing reflection is not perfect (red arrows in Fig. $8(\mathrm{~b})$ ), but the amplitude of the reflected wave is generally attenuated (blue arrow in Fig. 8(b)). Similar considerations apply also for the reflection associated with the wall of the skull below the focal plane, where again the coda of the focusing function (black arrows in Fig. 8) is shown to interfere destructively (blue arrows in Figs. 8(d) and $8(\mathrm{f})$ ) with the ingoing-reflection (red arrows in Figs. $8(\mathrm{c})$ and $8(\mathrm{e}))$.

The differences between the two discussed focusing strategies are visualized in an other way in Fig. 9, where the $L_{2}$ norm of the pressure wavefields associated with Eqs. 3 and 12 is plotted as a function of space. Note that both maps are normalized to allow proper comparison of the two focusing methods. In Standard Time-Reversal Focusing, the norm of the pressure wavefield exhibits a peak at the focal point (blue arrow in Fig. 9a), and significant values are almost homogeneously distributed throughout the brain (red arrows in Fig. 9a). This indicates that wave propagation occurs in the entire brain, which could be undesirable for medical treatments designed to target the focal point while not affecting other portions of the brain. The situation is rather different when focusing is achieved via solution of Eq. 12. Due to the peculiar focusing condition associated with Marchenko schemes ([11]), the corresponding wavefield still exhibits a peak at the focal point (blue arrow in Fig. 9(b)) while being mostly confined into a double cone centered at the focal point (blue cones in Fig. $9(\mathrm{~b})$ ). Yellow and green arrows point at regions of the brain with minimal wavefield propagation inside the brain and large amplitude spots outside the brain associated with the propagation of the coda of the focusing functions, respectively. The different performances of Focusing in Finite Time and Time-Reversal Focusing can be better appreciated in 10, where horizontal (a) and vertical (b) sections of the maps in 9 are plotted in logarithmic scale. As expected, along the horizontal section (a) Focusing in Finite Time exhibits reduced 
wavefield propagation, whereas along the vertical direction (b) the two diagrams are rather similar. Note that in Time-Reversal mirroring reflections occurr before and after time $t=0$, whereas in Focusing in Finite Time the interaction of the wavefield with the focal point theoretically occurs only at time $t=0$. Therefore, in Time-Reversal mirroring the norm of the wavefield at the focal point is intrinsically associated with both direct and scattered waves, while in Focusing in Finite Time it is theoretically only associated with direct components of the focusing functions $f$.

\section{Discussion}

The wavefields resulting from the time-reversal method, as formulated by Eqs. 2 and 3 have infinite support in time, which could be disadvantageous for various applications. On the other hand since the focusing functions $f_{1}$ and $f_{2}$ are confined in time and space by the direct propagation path from the boundary to the focal point [10], so is their superposition $f$. Hence, this focusing function seems preferable for wavefield focusing in finite time. As can be observed in Figs. 5, 7, and 8, the focusing function $f$ contains a series of wavefronts that are emitted into the medium from the surrounding boundary. Only the first of these wavefronts reaches the focal point. The remaining events are encoded such that any ingoing reflection of the first pulse is canceled or, when perfect focusing is not achieved, at least suppressed. Hence, the focusing function might be an attractive solution of the wave equation for focusing below strong acoustic contrasts. By canceling or reducing the amplitude of ingoing reflections, we achieve the desirable situation of a single wavefront or reduced energy to reach the focal point and propagate along the focal plane. Moreover, the peculiar nature of the focusing achieved by Eq. 12 minimizes the spatial exposure to the incident wavefield of the layer embedding the focal point, and this could possibly be beneficial for sensitivity analysis and/or safety concern in medical treatment [23]. Focusing functions associated with Eq. 12 may also therefore be useful input for inversion. Akin to Green's functions, they obey the wave equation, which can be inverted for the medium properties $c(\mathbf{x})$ and $\rho(\mathbf{x})$. In particular cases, they may be preferred over Green's functions for this purpose, since the entire signals can be captured by a concise recording in the time domain and exhibit peculiar sensitivity distributions. In the numerical tests considered here, we used either kinematically equivalent (first numerical experiment) or exact velocity models (second numerical experiment) to compute the initial focusing functions. When a poor background model is used, solutions from above and below could focus at different points, and the terms associated with the Green's functions in Eqs 6.7 and 10 11 would not cancel out, thus violating the focusing condition exhibited by $f$. Note that this restriction holds also for the time-reversal method when applied from two sides. Finally, in our numerical tests we have also neglected dissipation, which plays a key role in medical treatment and is critical in standard Marchenko applications with single-sided access media. However, when media are accessible from two sides (which is a strict requirement in the focusing strategy discussed in this paper), Marchenko redatuming can be adapted to account for dissipation [24. Future research will be devoted to extension of the proposed method to account for dissipative media.

\section{Conclusion}

A new integral representation has been derived for wavefield focusing in an acoustic medium. Unlike in the classical representation for this problem, which is based on time-reversed acoustics, the input and output signals for this type of focusing are finite in time. The method has been validated numerically for a head model consisting of hard (skull) and soft (brain) tissue, showing that it can lead to a reduction of spatial and temporal exposure when wavefield focusing is applied in practice.

\section{Acknowledgments}

This work is partly funded by the European Research Council (ERC) under the European Union's Horizon 2020 research and innovation programme (grant agreement No: 742703). 

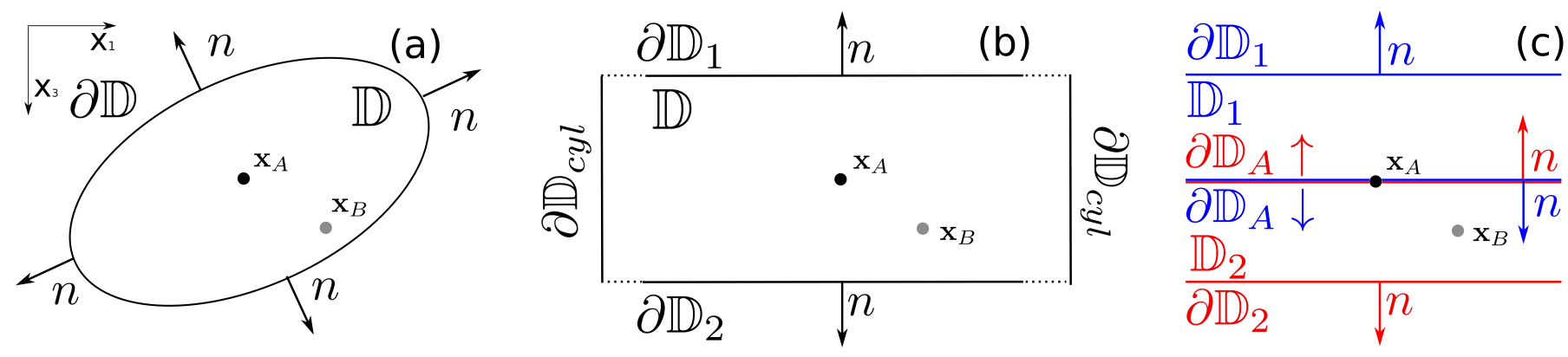

Figure 1: (a) Cross-section of the configuration in the $\left(x_{1}, x_{3}\right)$-plane for Eq. 2. Volume $\mathbb{D}$ is enclosed by $\partial \mathbb{D}$ (solid line) with outward-pointing normal vectors $n$. (b) Cross-section of the configuration for Eq. 3. Volume $\mathbb{D}$ is enclosed by $\partial \mathbb{D}_{1} \cup \partial \mathbb{D}_{2} \cup \partial \mathbb{D}_{\text {cyl }}$ (solid black lines). (c) Cross-section of the configuration for Eq. 12 Volume $\mathbb{D}$ is splitted into $\mathbb{D}_{1}$ and $\mathbb{D}_{2}$, surrounded by $\partial \mathbb{D}_{1} \cup \partial \mathbb{D}_{A}$ (blue line) and $\partial \mathbb{D}_{2} \cup \partial \mathbb{D}_{A}$ (red line), respectively. Note that the normals $n$ relative to $\partial \mathbb{D}_{1} \cup \partial \mathbb{D}_{A}$ and $\partial \mathbb{D}_{2} \cup \partial \mathbb{D}_{A}$ across $\partial \mathbb{D}_{A}$ are antiparallel. The focal point is at $\mathbf{x}_{\mathbf{A}} \in \partial \mathbb{D}_{\mathbf{A}}$.

\begin{tabular}{|l|l|l|}
\hline Tissue & velocity $(\mathrm{m} / \mathrm{s})$ & density $\left(\mathrm{kg} / \mathrm{m}^{3}\right)$ \\
\hline Muscle & 1588 & 1090 \\
\hline Skull & 2813 & 1908 \\
\hline Water & 1578 & 994 \\
\hline Blood & 1578 & 1050 \\
\hline Brain & 1546 & 1046 \\
\hline \hline
\end{tabular}

Table 1: Velocity and density values for the head model used in the second experiment (see Fig. 66.

\section{References}

[1] M. Fink, Journal of Physics D: Applied Physics, 26, 1333-1350 (1993).

[2] K. Wapenaar, E. Slob and R. Snieder, Physical Review Letters, 97, 234301 (2006).

[3] J. L. Thomas, F. Wu and M. Fink, Ultrasonic imaging, 18, 106-121 (1996).

[4] J. F. Aubry, M. Pernot, M. Tanter, G. Montaldo and M. Fink, Journal de Radiologie, 88, 1801-1809 (2007).

[5] S. Catheline, M. Fink, N. Quieffin and R. J. Ing, Applied Physics Letters, 90, 063902 (2007).

[6] Li, Z. and M. van der Baan, Geophysics, 81, JS123-KS134 (2016).

[7] G.A. McMechan, Geophysical Prospecting, 31, 413-420 (1983).

[8] M. K. Oristaglio, Inverse Problems, 5, 1097-1105 (1989).

[9] J. H. Rose, Inverse Problems 18, 1923-1934 (2002).

[10] R. Burridge, Wave Motion 2, 305-323 (1980).

[11] K. Wapenaar, and J. Thorbecke, Geophysical Prospecting, 65, 1430-1451 (2017).

[12] F. Broggini and R. Snieder, European Journal of Physics, 33, 593-613 (2012).

[13] K. Wapenaar, J. Thorbecke, J. van der Neut, F. Broggini, E. Slob, and R. Snieder, Journal of the Acoustical Society of America 135, 2847-2861 (2014).

[14] M. Ravasi, I. Vasconcelos, A. Kritski, A. Curtis, C. Alberto da Costa Filho, and G. Meles, Geophysical Journal International, 205, 99-104 (2016).

[15] K. Wapenaar, J. Thorbecke and J. van der Neut, Geophysical Journal International, 205, 531-535 (2016).

[16] J. T. Fokkema and P. M. van den Berg, Elsevier Science Publishing Company, Inc. (1993). 


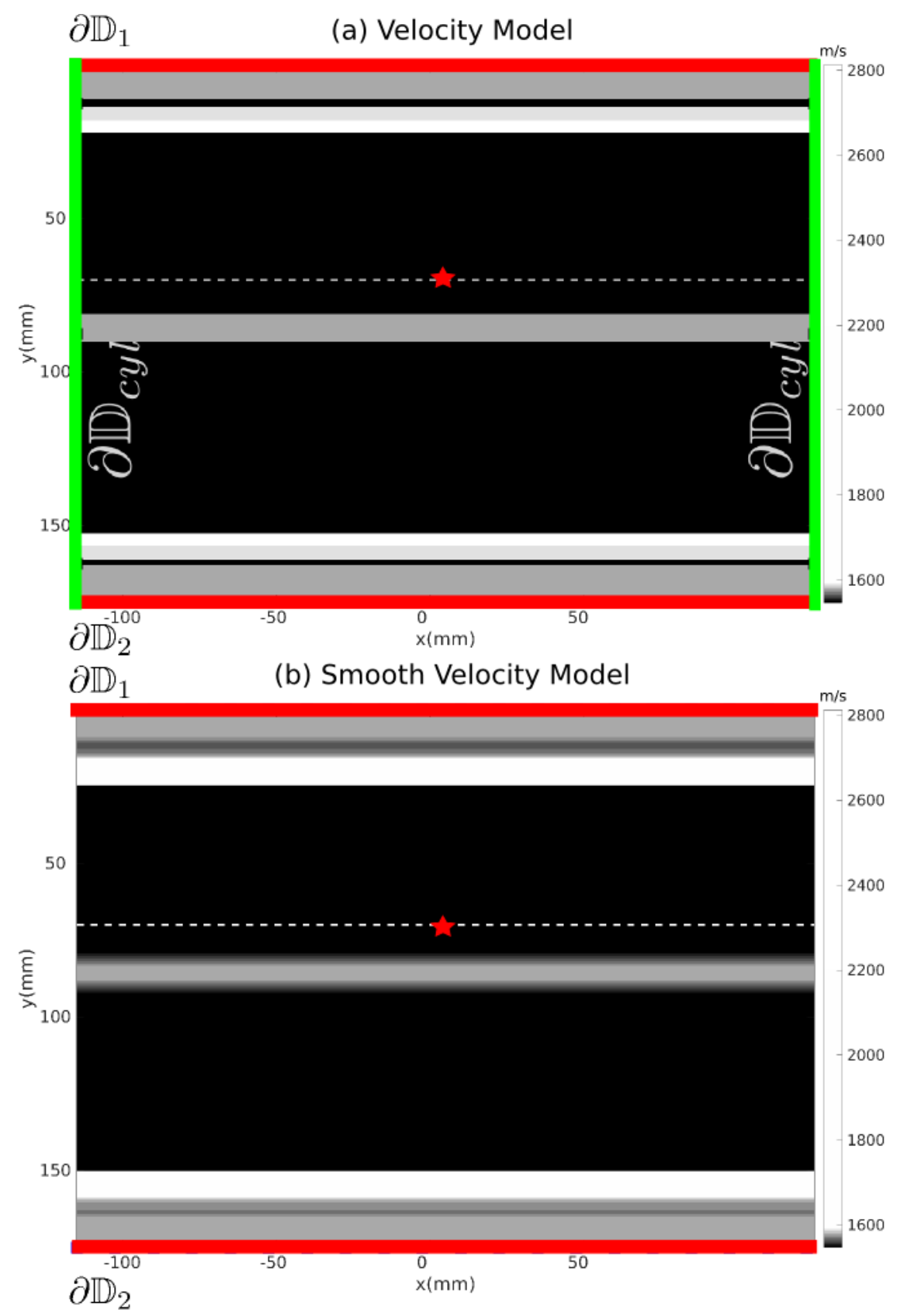

Figure 2: (a) True velocity model used in the first numerical experiment, corresponding to a 1D slice of a human head model (see Fig. 6 and Table 1). The red star and the dashed line represent the focusing point and plane, respectively. For the time-reversal focusing experiment associated with Eq. 2 (see Fig. 3), wavefields emanating from the focusing point and recorded at evenly sampled receivers distributed along a closed boundary $\partial \mathbb{D}_{1} \cup \partial \mathbb{D}_{2} \cup \partial \mathbb{D}_{c y l}$ (thick red and green lines) are used. For the time-reversal focusing experiment associated with Eq. 3 (see Fig. 4), only wavefields recorded along horizontal boundaries $\partial \mathbb{D}_{1} \cup \partial \mathbb{D}_{2}$ (thick red lines) are used. For the focusing experiment associated with Eq. 12 (see Fig. 5), a total of evenly sampled $481 \times 2$ co-located sources and receivers (indicated by the thick red lines) are used to compute reflection data along the upper $\left(\partial \mathbb{D}_{1}\right)$ and the lower $\left(\partial \mathbb{D}_{2}\right)$ horizontal boundaries. Standard Marchenko methods are employed to retrieve focusing functions $f_{1}$ and $f_{2}$ using reflection data associated with $\partial \mathbb{D}_{1}$ and $\partial \mathbb{D}_{1}$, respectively. [13. (b) Smooth velocity model used to compute the initial focusing function emanating from the focusing point (red star) and recorded along the upper $\left(\partial \mathbb{D}_{1}\right)$ and the lower $\left(\partial \mathbb{D}_{2}\right)$ horizontal boundaries (thick red lines). 

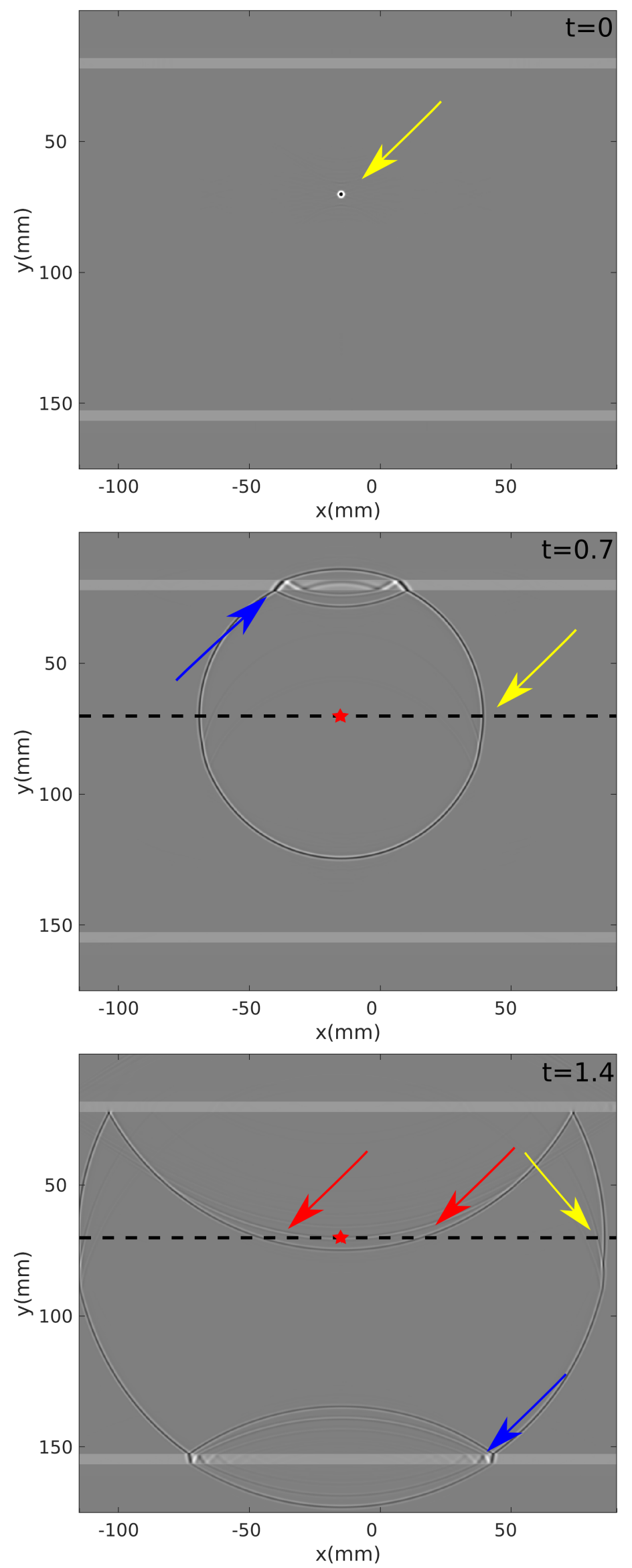

Figure 3: Snapshots of the time-reversed solution when a closed boundary is considered (Eq. 2). The focusing condition is satisfied, and the wavefield at time $t=0$ is perfectly isotropic (yellow arrow). At time $t>0$ direct (yellow arrows) as well as scattered (blue arrows) components of the wavefield are properly reconstructed. Red arrows indicate propagation of scattered waves through the focal plane. White lines indicates reflectors, shown here for interpretation only, while the red star and the black dashed line stand for the focal point and plane, respectively. For the sake of brevity only positive times are discussed, but identical considerations apply for the acausal components of the considered wavefield. 

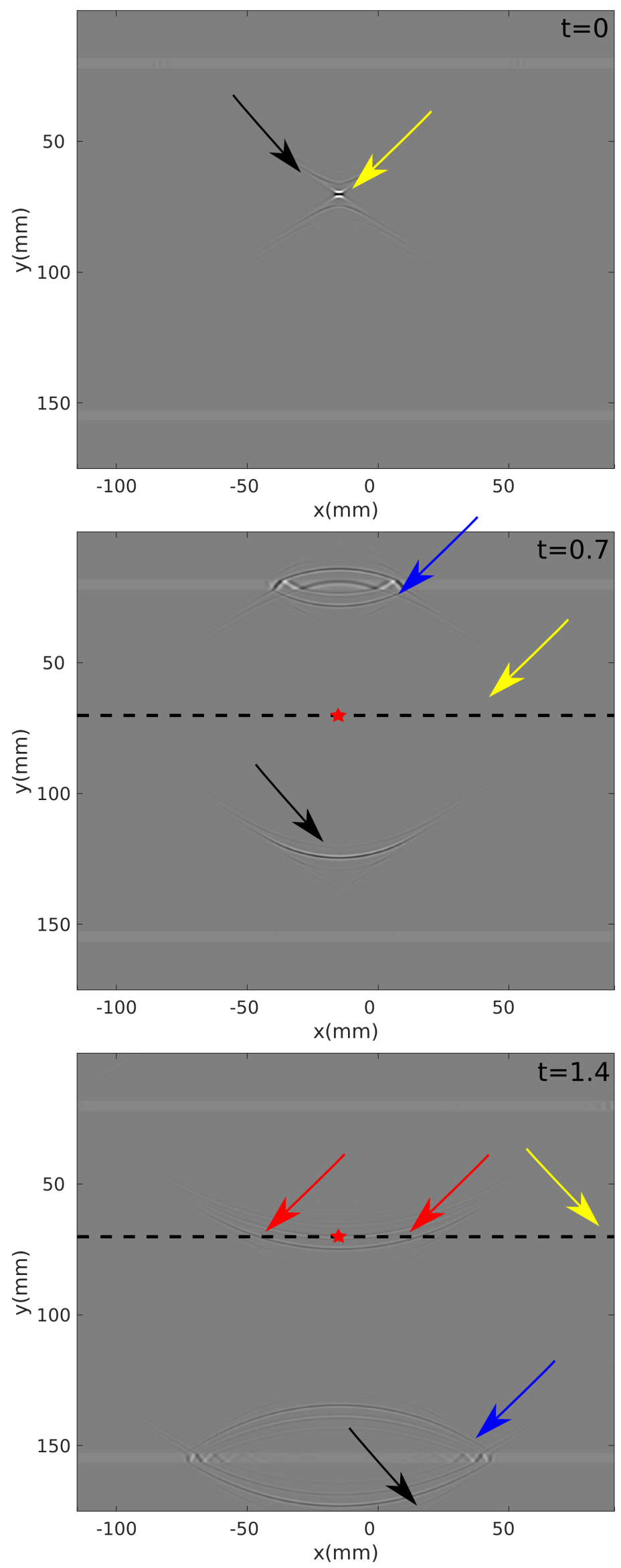

Figure 4: Snapshots of the time-reversed solution when partial boundaries are considered (Eq. 3). Due to the finite extent of the injection boundaries $\partial \mathbb{D}_{1}$ and $\partial \mathbb{D}_{2}$, the wavefield at time $t=0$ is not perfectly isotropic (yellow arrow), and artefacts, with maximum amplitude $\sim 5 \%$ of the focus magnitude, contaminate the wavefield throughout the entire simulation (black arrows). At times $t>0$ scattered components of the wavefield are relatively well reconstructed (blue arrows), but the direct component of the wavefield exhibits distorted amplitude along the horizontal direction (yellow arrows). Red arrows indicate propagation of scattered waves through the focal plane. Keys as in Fig. 3. 

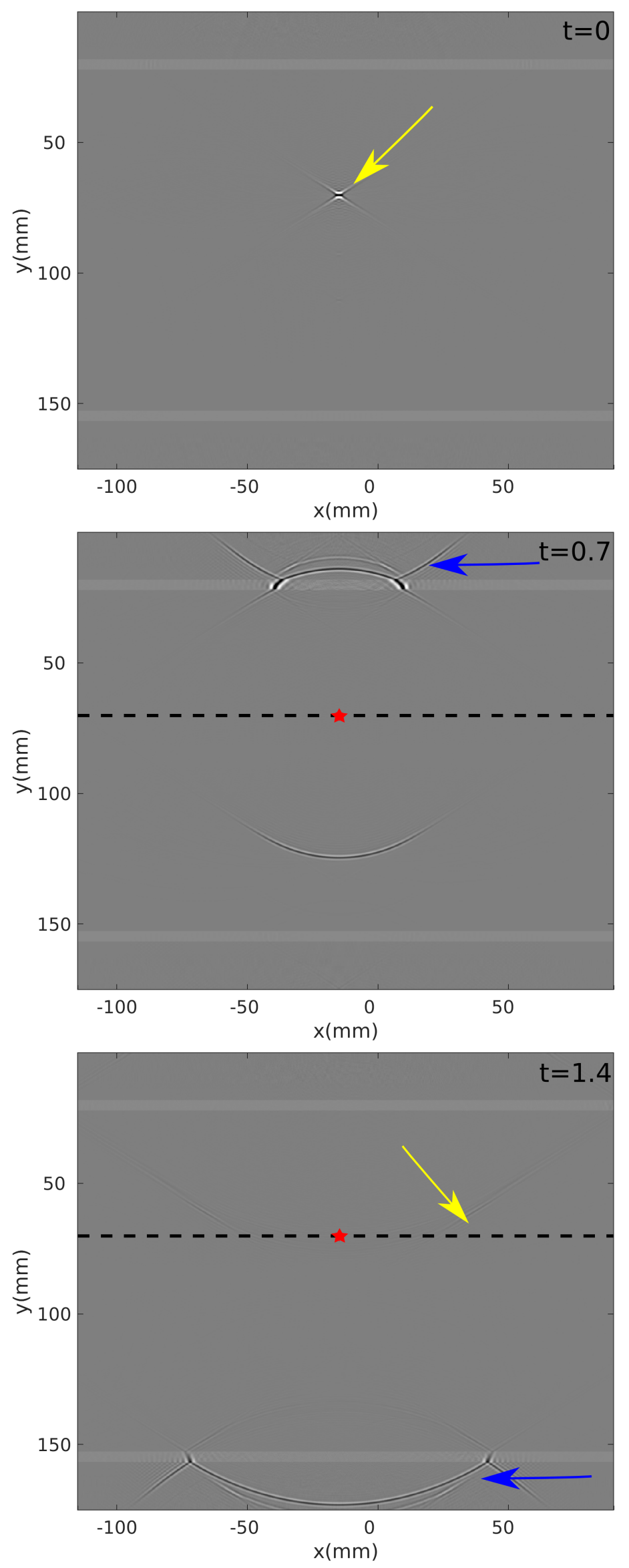

Figure 5: Snapshots of the focusing in finite time with minimal spatial exposure solution (Eq. 12). The focusing condition is satisfied except that for low amplitude artefacts, with amplitude $\sim 2 \%$ of the focus magnitude, propagating along the focal plane at times $t>0$ (yellow arrows). Note that the wavefield at time $t=0$ is not supposed to be vanishing throughout the domain (black arrows indicate propagation of the coda of $f$ ). At times $t>0$ scattered (blue arrows) components of the wavefield are suppressed by destructive interference with propagation of the coda of $f$. The green shaded area indicates the layer embedding the focal point, where only direct components of the focusing function $f$ propagate. Keys as in Fig. 3. 

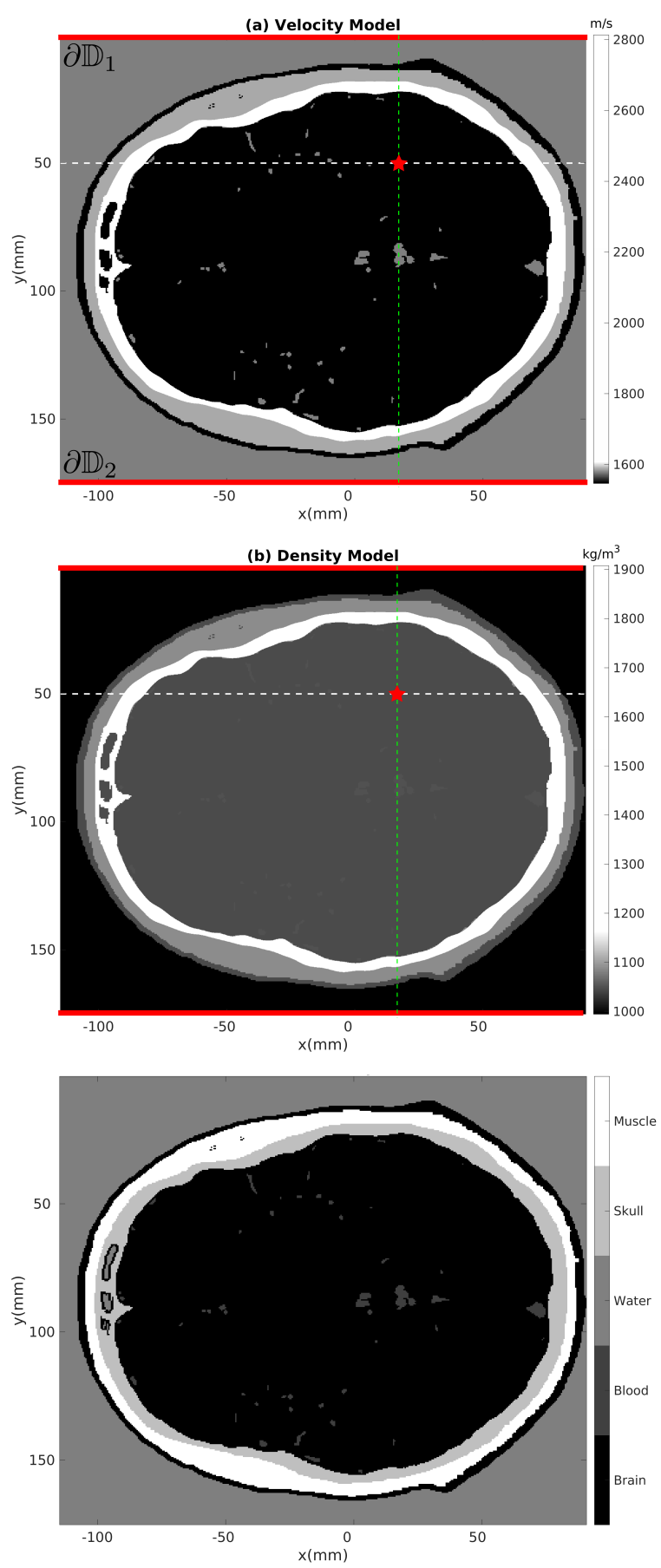

Figure 6: (a) True velocity model used in the second numerical experiment. The red star and the gray dashed line represent the focusing point and plane, respectively. The green line indicates the 1D profile used for the first numerical experiment. For the time-reversal focusing experiment associated with Eq. 3 (see left columns of Figs. 7 and 8), wavefields emanating from the focusing point and recorded at evenly spaced receivers located along horizontal boundaries $\partial \mathbb{D}_{1} \cup \partial \mathbb{D}_{2}$ (thick red lines) are used. For the focusing experiment associated with Eq. 12 ((see right columns of Figs. 7 and 80$)$ ), a total of $481 \times 2$ evenly sampled co-located sources and receivers (thick red lines) are used to compute reflection data along the upper $\left(\partial \mathbb{D}_{1}\right)$ and the lower $\left(\partial \mathbb{D}_{2}\right)$ horizontal boundaries. Standard Marchenko methods are employed to retrieve focusing functions $f_{1}$ and $f_{2}$ using reflection data associated with $\partial \mathbb{D}_{1}$ and $\partial \mathbb{D}_{2}$, respectively. This velocity model is also used to compute the initial focusing function emanating from the focusing point (red star) and recorded along the upper $\left(\partial \mathbb{D}_{1}\right)$ and the lower $\left(\partial \mathbb{D}_{2}\right)$ horizontal boundaries (thick red lines). (b) True density model used in the second numerical experiments. (c) Anatomy of the brain used in the second numerical experiment. Keys as for (a). 

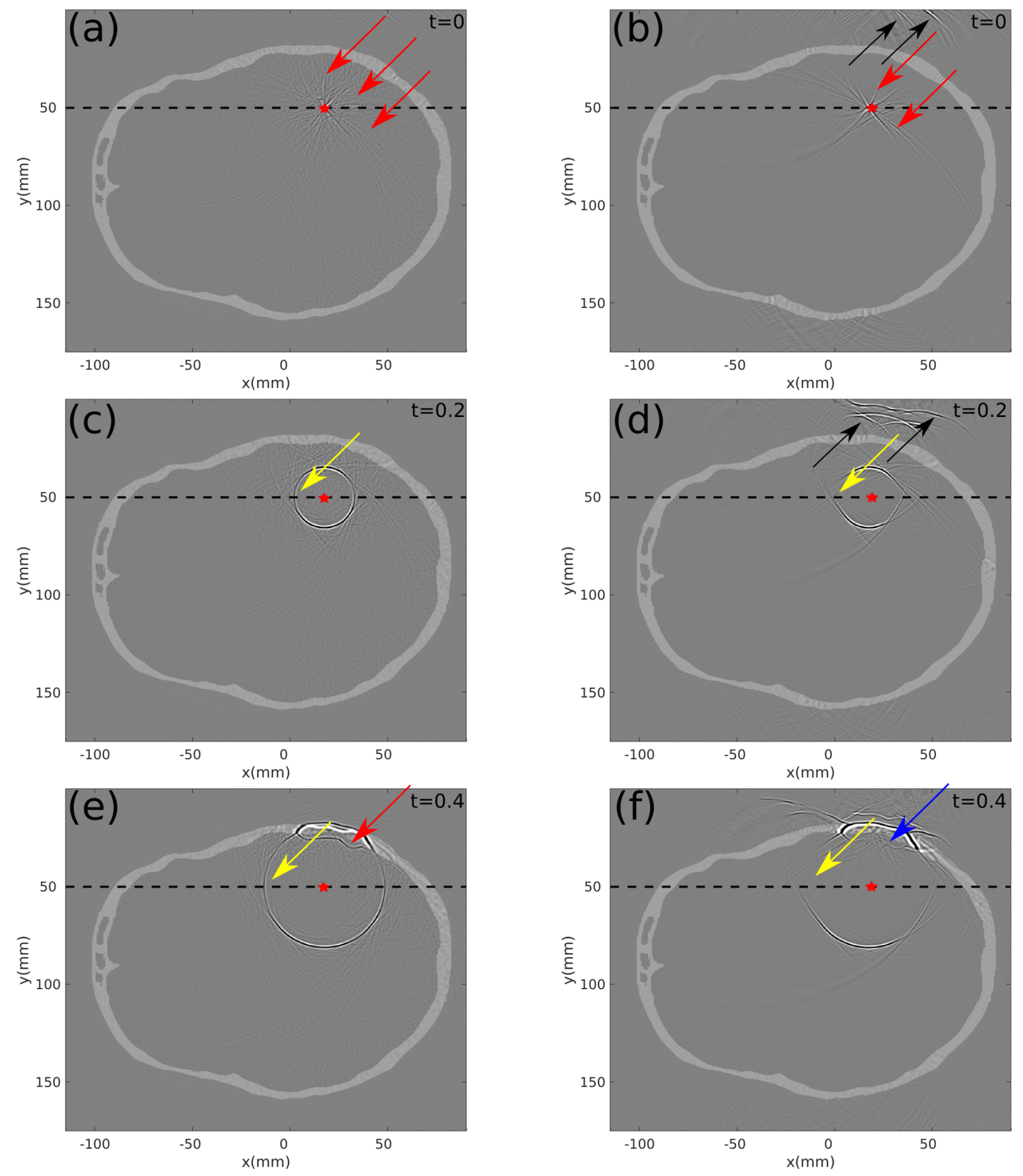

Figure 7: Left column: Snapshots of the time-reversed solution when partial boundaries are considered (Eq. 3). Due to the finite extent of the injection boundaries $\partial \mathbb{D}_{1}$ and $\mathbb{D}_{2}$, small amplitude artefacts contaminate the wavefield at time $t=0$ (red arrow in (a)). Due to the strong lateral reflections, at times $t>0$ direct components of the wavefield are relatively well reconstructed (yellow arrows in (c) and (e)). The red arrow in (e) indicates a scattered wave reflected at the interface above the focal plane. Right column: Snapshots of the focusing in finite time with minimal spatial exposure solution (Eq. 12). The focusing condition is satisfied except that for low amplitude artefacts, contaminating the domain at time $t=0$ (red arrow in (b)). Note that the wavefield at time $t=0$ is not supposed to be vanishing throughout the domain (black arrows indicate propagation of the coda of $f$ ). At times $t>0$ scattered components of the wavefield are attenuated by destructive interference with propagation of the coda of $f$ (blue arrow in (f)). Keys as in Fig. 3. 

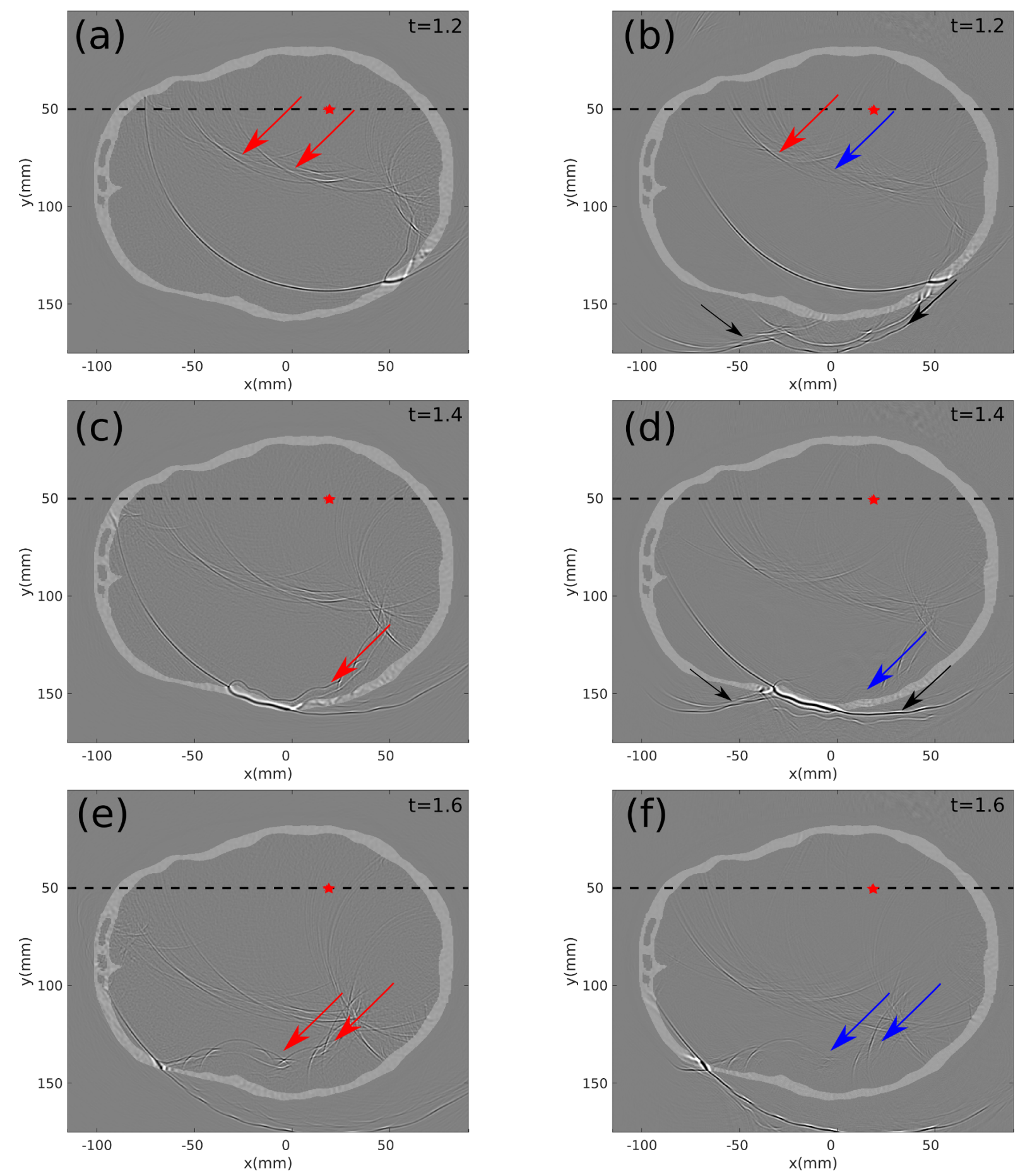

Figure 8: Left column: Snapshots of the time-reversed solution when partial boundaries are considered (Eq. 33. Red arrows point at reflections with the skull walls. Right column: Snapshots of the focusing in finite time with minimal spatial exposure solution (Eq. 12). Black and blue arrows point at the coda of the focusing functions and attenuated reflections, respectively. Keys as in Fig. 3. 


\section{(a) Time-Reversal Focusing}



(b) Finite Time Focusing

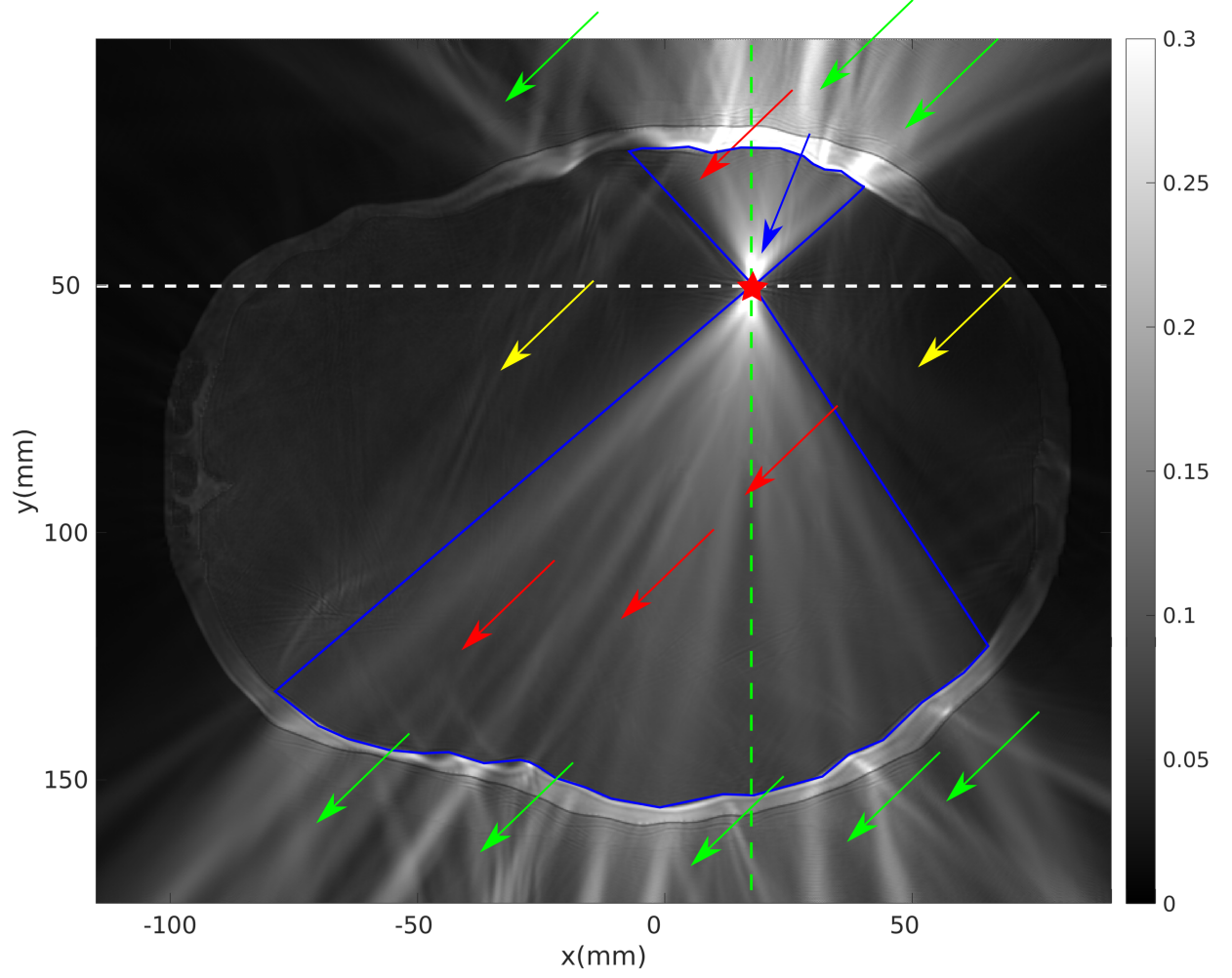

Figure 9: Normalized $L_{2}$ norm of the pressure wavefields associated with the left hand sides of Eqs. 3 (a) and 12 (b), respectively, plotted as functions of space. In Standard Time-Reversal focusing (a), the norm of the pressure wavefield exhibits a peak at the focal point (blue arrow in a), and significant values are almost homogeneously distributed throughout the model (red arrows in (a)). In finite time focusing, the wavefield is still exhibiting a peak at the focal point (blue arrow in Fig. (b)) while being somehow confined into a double cone centered at the focal point (blue cones in (b)). Yellow and green arrows point at regions of the brain with minimal wavefield propagation and large amplitude spots associated with the propagation of the coda of the focusing functions, respectively. Gray and green dashed lines indicate horizontal and vertical sections used in Fig. 10, respectively. Keys as in Fig. 6. 
(a) Horizontal Slice

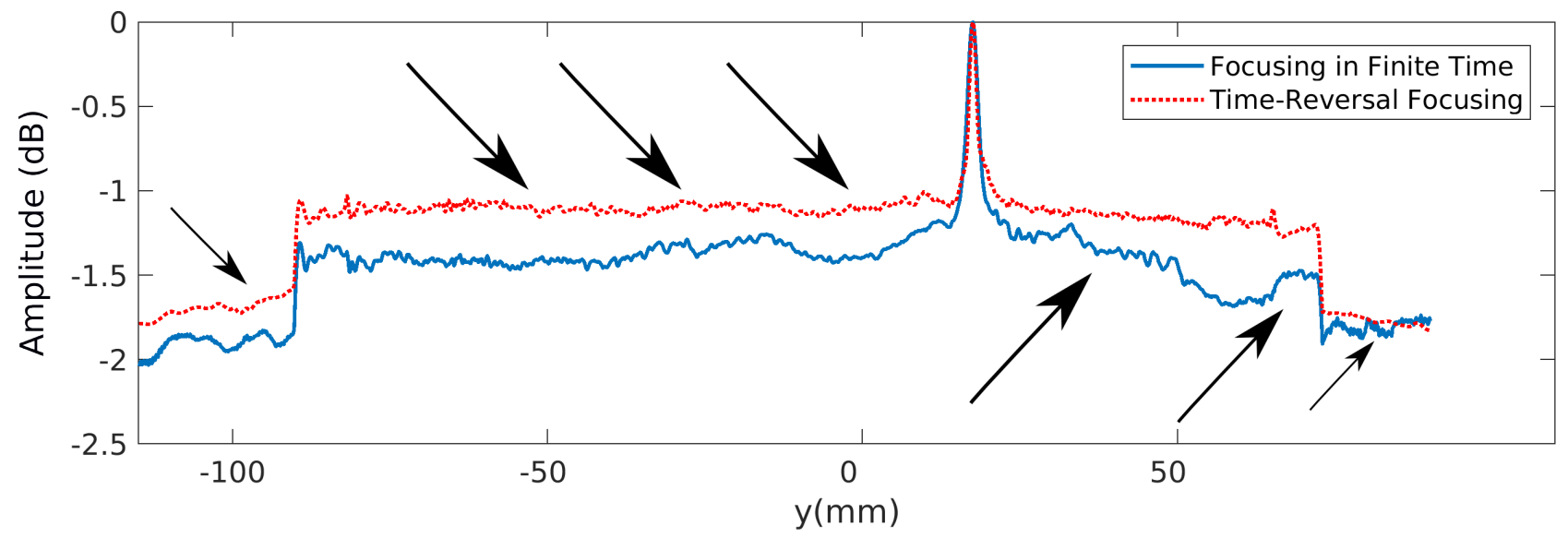

(b) Vertical Slice

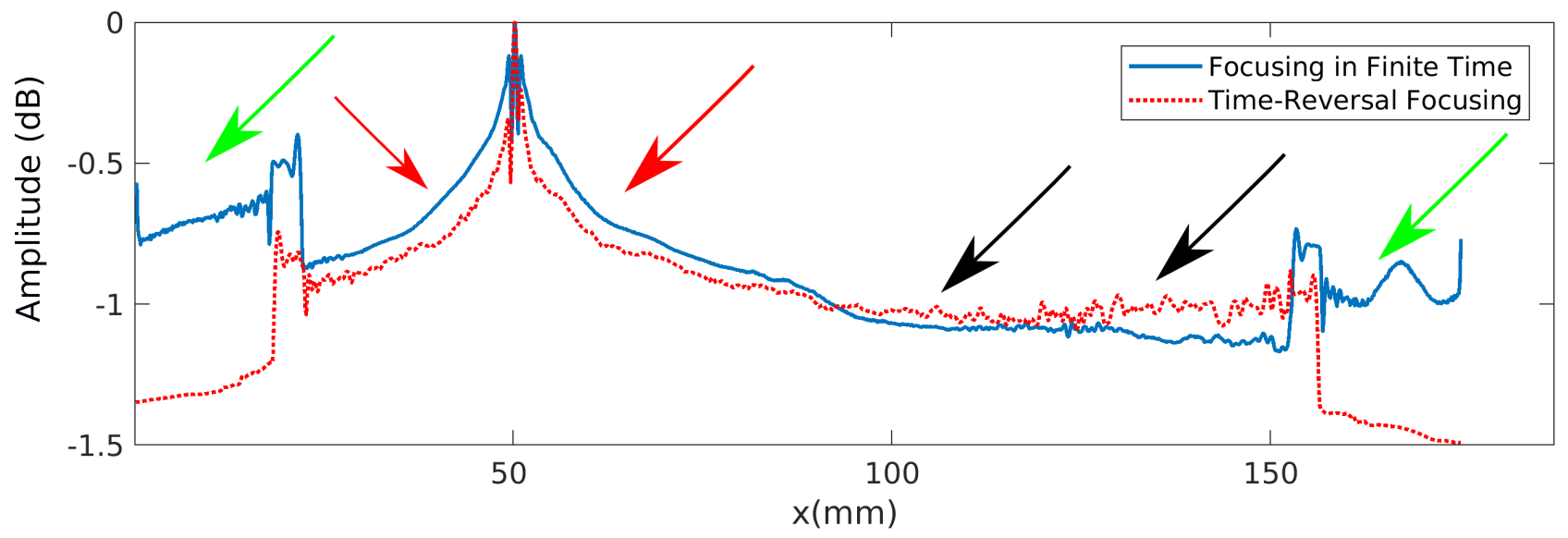

Figure 10: Horizontal (a) and vertical (b) slices of the maps in Fig. 9, plotted in logarithmic scale. Black arrows in (a) indicate large portions of the focal plane (gray dashed lines in Fig. 9) where wavefield propagation in Focusing in Finite Time is significantly reduced as opposed to Time-Reversal Focusing. The red and black arrows in (b) indicate zones along the green dashed lines in in Fig. 99 where Focusing in Finite Time and Time-Reversal Focusing involve slightly large and slightly smaller wavefield propagation, respectively. Green arrows point at zones outside of the brain where Focusing in Finite Time involve propagation of coda exhibiting large amplitudes (see green arrows in Fig. 9(b)). 
[17] K. Wapenaar, J. Thorbecke, J. van der Neut, E. Slob and R. Snieder, Geophysical Prospecting, 65, 14111429 (2017).

[18] K. Wapenaar,, J. Thorbecke, J. van der Neut, Geophysical Journal International, 205, 531-535 (2016).

[19] L. Fishman, Radio Science, 28, 865-876 (1993)

[20] N. N. Bojarski, Journal of the Acoustical Society of America, 74, 281-285 (1983).

[21] J. Thorbecke, E. Slob, J. Brackenhoff, J. van der Neut and K. Wapenaar, Geophysics, 82, WB29-WB45 (2017).

[22] R. Snieder, K. Wapenaar, K. Larner, Geophysics, 71, SI111-SI124 (2006).

[23] A. Hughes, K. Hynynen, Physics in Medicine \& Biology, 17, L9-L19 (2017).

[24] E. Slob, Physical Review Letters, 116, 164301-1-164301-6 (2016). 